\title{
Hemodynamic analysis and implantation strategies of delayed intracranial aneurysm rupture after flow diverter treatment
}

\author{
Shiyao Chen ${ }^{1}$, Bin $\mathrm{Bai}^{1}$, Nan $\mathrm{Lv}^{2}$, Yunzhang Cheng ${ }^{1}$, Bin $\mathrm{Ji}^{3}$ \\ ${ }^{1}$ Shanghai Interventional Medical Device Engineering Technology Research Center, University of Shanghai for Science and Technology, Shanghai, \\ China; ${ }^{2}$ Department of Neurosurgery, First Affiliated Hospital of Naval Military Medical University, Changhai Hospital of Shanghai, Shanghai, \\ China; ${ }^{3}$ Department of Radiology, Shanghai East Hospital, School of Medicine, Tongji University, Shanghai, China \\ Contributions: (I) Conception and design: S Chen, B Bai; (II) Administrative support: Y Cheng, B Ji; (III) Provision of study materials or patients: N \\ Lv; (IV) Collection and assembly of data: S Chen; (V) Data analysis and interpretation: S Chen; (VI) Manuscript writing: All authors; (VII) Final \\ approval of manuscript: All authors. \\ Correspondence to: Bin Ji. Department of Radiology, Shanghai East Hospital, School of Medicine, Tongji University, 150 Jimo Road, Shanghai 200120 , \\ China. Email: jibin1017@sina.com.
}

Background: Delayed aneurysm rupture after flow diverters (FDs) is a serious complication which mechanism remains unclear. The hemodynamics of FDs with proximal or distal densification implantation strategies have rarely been reported. In this study, we investigated not only the hemodynamic factors involved in postoperative rupture, but also the hemodynamic effects of different FDs implantation strategies on avoiding this complication.

Methods: We selected 2 internal carotid artery (ICA) aneurysms with similar morphological characteristics, both of which were treated with FDs but had opposite therapeutic outcomes (Case 1, ruptured after FD treatment; Case 2, recovered). The FDs strategies we designed were strategy A [with homogeneous 30\% metal coverage ratio (MCR)], strategy B (with distal densification of $40 \%$ and proximal $30 \% \mathrm{MCR}$ ) and strategy C (with proximal densification of $40 \%$ and distal $30 \%$ MCR). Virtually FDs deployment and computational fluid dynamics (CFD) method were performed to simulate FDs implantation strategies and analyze the hemodynamics associated with postoperative rupture.

Results: After FDs implantation, the velocity of blood entering the aneurysm decreased (Case 1, 25.4\%; Case 2, 30.6\%), but the inflow jet impingement still existed in Case 1. The overall WSS decreased similarly in both cases, but the high WSS region hardly diminished in Case 1. For overall wall pressure, Case 2 decreased slightly but increased in Case 1 . Of the three FDs implantation strategies, strategy $\mathrm{C}$ had the best hemodynamic effects, including the maximum blood velocity reduction and a tendency to form a more stable flow pattern, the maximum reduction rate of overall WSS and the effective diminish of high WSS area as well as the overall decrease of wall pressure.

Conclusions: Not significant decrease of blood flow velocity entering the aneurysm adding persistent impact of inflow jet impingement, high WSS area that did not diminish and abnormal increase of pressure on the aneurysm wall may be causative of postoperative rupture and bleeding of ICA aneurysms. In addition, the hemodynamic effects were favorable when the FD was improved to proximal densification, which may reduce the risk of delayed aneurysm rupture following FDs treatment.

Keywords: Delayed rupture; flow diverter (FD); computational fluid dynamics (CFD); intracranial aneurysm (IAs); internal carotid artery (ICA)

Submitted Oct 15, 2021. Accepted for publication Dec 02, 2021.

doi: 10.21037/atm-21-5939

View this article at: https://dx.doi.org/10.21037/atm-21-5939 


\section{Introduction}

The rupture of intracranial aneurysms (IAs) is the primary cause of subarachnoid hemorrhage (SAH) with high morbidity and mortality (1). Although the natural history of IAs is still lack of understanding, the importance of hemodynamics in IAs rupture has been established (2). Flow diverters (FDs), as a new interventional method, with the aim of promoting intra-aneurysmal thrombosis by altering the hemodynamic environment of the aneurysm lumen and its parent artery, have significantly improved the long-term efficacy of interventional treatment of the aneurysm $(3,4)$. FDs were initially used to treat the large or giant wide-necked IAs located at the internal carotid artery (ICA) proximal to the posterior communicating artery (5). According to related literature reports, the aneurysmal complete occlusion rates can be up to $90.8-$ $95 \%$ at 12 -month follow-up after FDs placement $(6,7)$. Nevertheless, with the increasingly widespread application of FDs, several reports suggested that attention should be paid to the complications following FDs treatments carrying an overall incidence of $17 \%$ and perioperative fatality can be $2.8-4 \%(8-10)$. Delayed aneurysm rupture is one of the most serious complications after FDs treatment, often leading to SAH or carotid-cavernous fistula (11). The occurrence of postoperative rupture was about $4.0 \%$ (7), and the prognosis was poor with mortality or neurological dysfunction rate of $81.3 \%$ (12). Several studies were conducted on the hemodynamic risk factors associated with delayed aneurysm rupture (13-15), but the underlying mechanism of delayed rupture remains uncertain.

The aneurysm occlusion cannot complete immediately after FDs implantation, which means the aneurysm is still at risk of rupture until complete embolization. Several large clinical studies have supported the FDs implantation with different metal coverage ratio (MCR) have different effects on the hemodynamics in IAs treatment (16-19). FDs implantation strategies with low MCR may not form complete aneurysmal embolization, while FDs with high MCR can significantly guide the blood along the axis of the parent artery which also may increase the pressure of the aneurysms by overlapping multiple FDs to have higher rupture risk of IAs (20). Nonetheless, previous studies mainly focused on the effects of FDs with homogeneous densification, the hemodynamic effect of proximal or distal densification of FDs has not been studied.

Sidewall ICA aneurysms have comparatively low annual rupture risk of $0.2 \%$, but account for nearly $40 \%$ of all aneurysm cases (21). Compared with other sites, ICA aneurysms have higher growth risk and tend to cause compression of surrounding tissues due to the proximity to cranial nerve or brain structures that make it difficult to perform surgical access at this location (22). So in this study, we selected two cases of sidewall ICA aneurysms, one of which had postoperative bleeding and the other had no postoperative complications. Virtual FDs placement technology was used to reproduce the treatment process, and computational fluid dynamics (CFD) method was performed to compare the difference of hemodynamic characteristics between the two cases. Besides, we also reconstructed two additional FDs with distal or proximal densification, to analyze the hemodynamic effects of the different FDs implantation strategies. The aim of this study was to improve the understanding of hemodynamic changes after FDs therapy.

We present the following article in accordance with the MDAR reporting checklist (available at https://dx.doi. org/10.21037/atm-21-5939).

\section{Methods}

The study was conducted in accordance with the Declaration of Helsinki (as revised in 2013). This retrospective study was approved by the Institution Review Board of Changhai Hospital. The requirement for informed consent was waived for this retrospective study and the patients' information was anonymized prior to analysis.

\section{Patient-specific aneurysm models}

We selected 2 cases of large ICA aneurysms treated with FDs. The 3D patient-specific aneurysm models were extracted by digital subtraction angiography (DSA) images, where Case 1 presented a fatal aneurysm rupture after FD placement, while Case 2 had no postoperative complications. The aneurysm models were imported into Geomagic Studio 2015 (3D Systems, Rock Hill, SC, USA) for repair, segmentation, smoothing, thinning, and so on, and then stored in STL format. Table 1 indicates the morphological parameters of the 2 cases.

The antiplatelet aggregation protocol is as follows. The patients were routinely treated with aspirin of $300 \mathrm{mg}$ and clopidogrel of $75 \mathrm{mg}$ once a day, for at least 3 days before FDs treatment. Systemic heparinization was performed during the operation, and heparin was naturally neutralized after the operation. After FDs implantation, 
Table 1 Morphological parameters of the ICA aneurysms

\begin{tabular}{lccc}
\hline Parameter & Case 1 & Case 2 & Difference \\
\hline Size $(\mathrm{mm})$ & 11.28 & 11.52 & -0.24 \\
Neck size $(\mathrm{mm})$ & 9.15 & 8.17 & 0.98 \\
AR & 1.23 & 1.41 & -0.18 \\
SR & 2.07 & 2.32 & -0.25 \\
V $\left(\mathrm{mm}^{3}\right)$ & $1,097.17$ & 575.24 & 521.93 \\
UI & 0.006 & 0.009 & -0.003 \\
EI & 0.137 & 0.107 & 0.03 \\
NSI & 0.139 & 0.109 & 0.03 \\
\hline
\end{tabular}

ICA, internal carotid artery; AR, aspect ratio; SR, size ratio; V, volume; UI, undulation index; El, ellipticity index; NSI, non-sphericity index.

the administration of aspirin of $300 \mathrm{mg}$ and clopidogrel of $75 \mathrm{mg}$ once a day was needed for 6 weeks. Clopidogrel treatment can be discontinued after 3 months, and aspirin of $300 \mathrm{mg}$ once a day was should be continued which was recommended for life.

\section{Virtual stent deployment and meshing}

FD with a wire diameter of $0.05 \mathrm{~mm}$ was constructed by SolidWorks (SolidWorks Corp., Waltham, MA, USA), and a total of three types of FDs with different MCR were established, as shown in Figure 1. First, the FDs with MCR of $30 \%$ (strategy A) were placed into these 2 IAs using virtual stent implantation technology to analyze the difference of hemodynamic parameters related to delayed aneurysm rupture before and after FD treatment. Next, the additional two FDs with different MCR (strategy B, FD with proximal MCR of $30 \%$ and distal MCR of $40 \%$; strategy C, FD with proximal MCR of $40 \%$ and distal MCR of $30 \%$ ) were implanted into the aneurysm model with delayed rupture to analyze the hemodynamic effect of different FDs strategies. The FDs deployed into the parent artery at the neck of the aneurysm models were illustrated in Figure 2.

After the deployment was completed, the aneurysm model containing the FD was imported in ANSYS ICEM CFD 18.0 (Ansys, Inc., Canonsburg, PA, USA) to create the mesh. Because of the huge magnitude difference between the FD wire diameter and the size of the aneurysm, to save calculation time, only the FD covering the neck of the aneurysm was retained. The maximum element size was set at $0.5 \mathrm{~mm}$ in the aneurysm, and $0.03 \mathrm{~mm}$ was set to the maximum element size near the metal struts of the FD to capture the boundary. The numbers and the quality of elements in the computational meshes for the aneurysm model and the model of the aneurysm with FD implantation are demonstrated in Table 2. The element number per cubic millimeter was more than 1,800 , which could verify that the computational results did not depend on the meshes (23).

\section{Boundary conditions and hemodynamic parameters}

The simulation was performed by ANSYS Fluent 18.0 (Ansys, Inc., USA). The governing equations underlying the calculation were the Navier-Stokes formulation. According to the calculation method of Reynolds number, the Reynolds number in this study was lower than 2,300, we treated blood as a Newtonian fluid (viscosity $\mu$ was $0.0035 \mathrm{~Pa} \cdot \mathrm{s})$, with an assumption of laminar and incompressible (density $\rho$ was $1,050 \mathrm{~kg} / \mathrm{m}^{3}$ ). The vessel was assumed to be rigid with non-slip boundary conditions, and the outlet was set to 0 static pressure (24). Ultrasound Doppler technology was utilized to collect the normal ICA pulsatile velocity profile. After simplified fitting by Matlab 2016a (MathWorks, Natick, MA, USA), the velocity curve was fixed as the inlet boundary condition through user-defined function (UDF) programming. The calculation adopted the simple algorithm, which was carried out in the second-order upwind formula, $1 \times 10^{-5}$ was set as the convergence accuracy, and we discretized the whole cardiac cycle of $0.8 \mathrm{~s}$ at a timestep of $0.001 \mathrm{~s}$ for numeric simulation. A total of 3 cardiac cycles were simulated to ensure a full periodicity, and the last cycle was taken as output. The hemodynamic parameters such as streamline, blood flow velocity, aneurysm pressure, wall shear stress (WSS), and other hemodynamic parameters at peak systole before and after stent deployment were compared. The average and maximum values of the blood 
Page 4 of 12

Strategy A

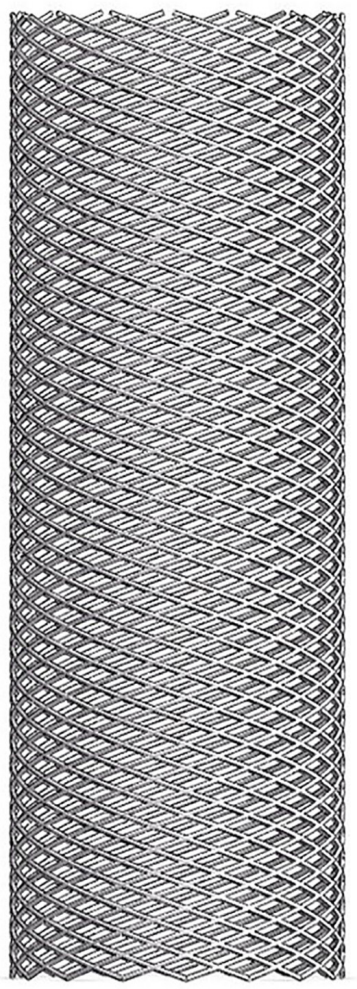

Chen et al. Hemodynamics and FDs implantation of delayed aneurysm rupture

Strategy B

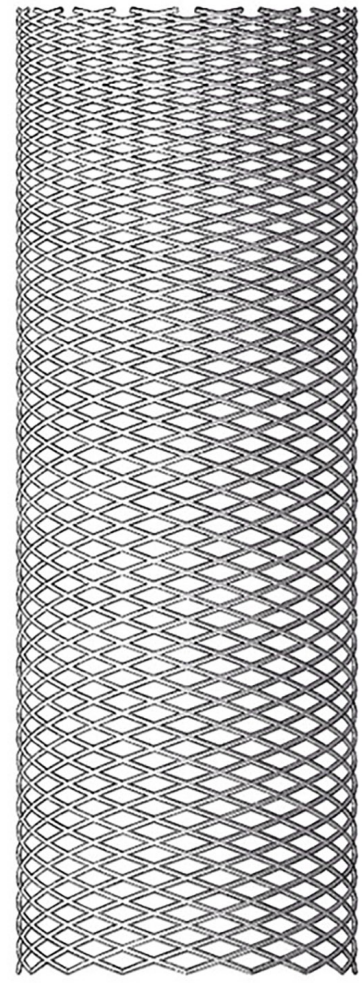

Strategy C

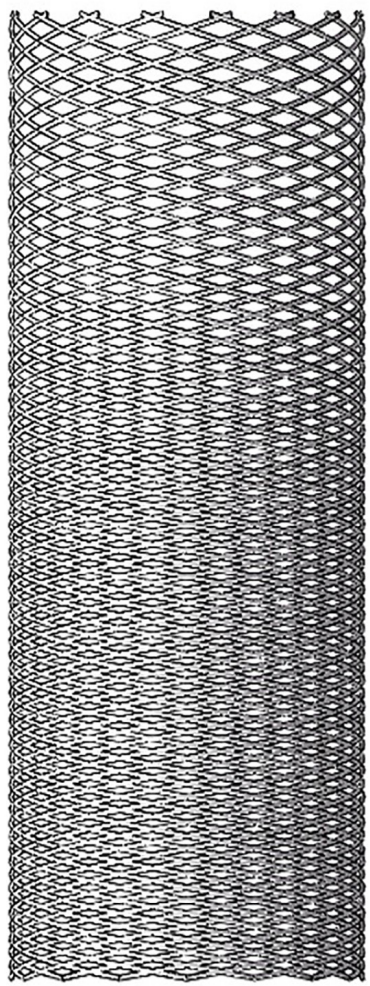

Figure 1 Three types of flow diverters (FDs) implantation strategies designed in this study. Strategy A, FDs with homogeneous $30 \%$ metal coverage ratio (MCR); strategy B, FDs with distal MCR of $40 \%$ and proximal MCR of 30\%; strategy C, FDs with distal MCR of $30 \%$ and proximal MCR of $40 \%$.

A

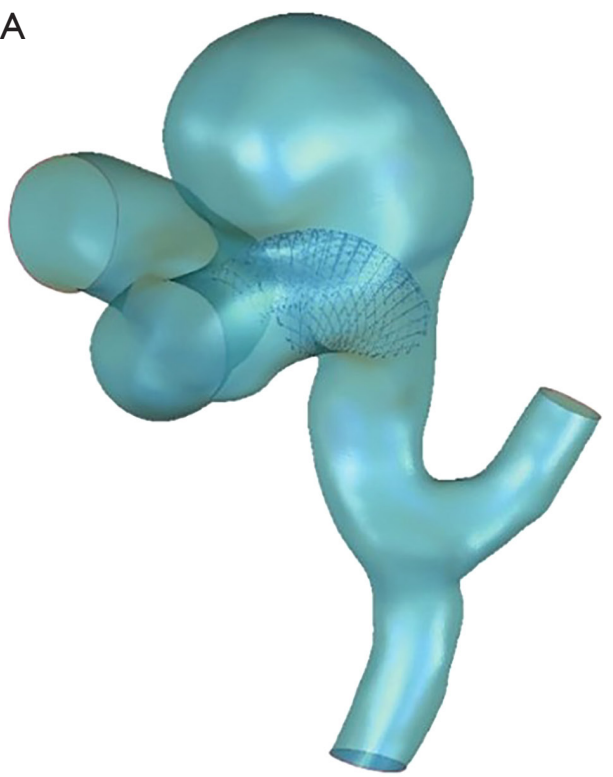

B

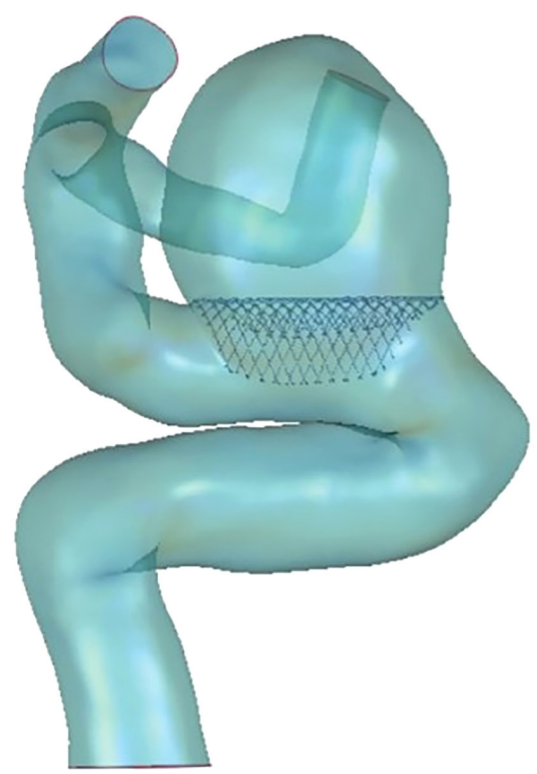

Figure 2 The placement and release of flow diverter (FD). (A) Case 1: delayed rupture; (B) Case 2: recovered. 
Table 2 Computational meshes before and after flow diverter implantation

\begin{tabular}{lcc}
\hline Case \# & Number of elements & Mesh quality \\
\hline Case 1 & & \\
Before & 270,000 & 0.39 \\
After & $5,080,000$ & 0.21 \\
Case 2 & \\
Before & 349,000 & 0.39 \\
After & $1,140,000$ & 0.30 \\
\hline
\end{tabular}

flow velocity were determined on the aneurysm neck plane and calculated at the peak systole.

\section{Statistical analysis}

The reduction ratio was calculated to compare the differences between before FDs treatment and after FDs treatment. The histograms were used to compare the reduction ratio of each hemodynamic parameter in two cases and three strategies.

\section{Results}

\section{Hemodynamics of IAs after FDs implantation}

In this part, we implanted FD with 30\% MCR virtually on the 2 large ICA aneurysms both of which were similar in morphology to analyze the difference of hemodynamic parameters related to delayed aneurysm rupture before and after FD treatment.

Streamlines, iso-surface velocity (ISO) of $0.4 \mathrm{~m} / \mathrm{s}$, WSS, and the pressure of aneurysm wall before and after the FD implantation for 2 cases are shown in Figures 3, respectively. Figure 4 shows the change of inflow volume, intra-aneurysmal pressure, the pressure on the aneurysmal wall, and time-averaged wall shear stress (AWSS) before and after the FD implantation for the 2 cases.

\section{Velocity and volume flow of blood}

In Case 1, blood flows into the aneurysm sac from the distal end of the parent artery, and then flows out of the middle of the aneurysm, forming a vortex-like flow pattern inside the aneurysm. It is also demonstrated that continuous inflow jet impingement on the distal side of the aneurysmal wall and aneurysm dome, with large volume flow and complex flow patterns in the aneurysm before
FD implantation. After FD placement, the volume flow of blood entering the aneurysm decreased slightly, but constant inflow jet impingement to the aneurysm dome is still existed. In Case 2, the intra-aneurysmal flow pattern before FD implantation is comparable to that of Case 1 . After FD placement, the blood flow velocity decreased, and the inflow jet impingement on the aneurysm dome disappeared. The change of velocity before and after the FD implantation was calculated to quantitatively evaluate the effect of FD treatment, and the decrease of aneurysmaveraged velocity of Case 1 and Case 2 was $25.38 \%$ and $30.56 \%$, respectively.

\section{WSS}

The overall aneurysm WSS decreased and the highest WSS values of aneurysmal walls changed from proximal to the distal aneurysmal neck after FD implantation in both cases. However, the high WSS region of the aneurysm dome previously still existed after FD implantation in Case 1, while the high WSS region of the aneurysm dome almost diminished. The WSS on the parent artery increased, with the maximum value at the outlet. The AWSS of the aneurysm surface was calculated. For Case 1 and Case 2, the AWSS decreased by $48.7 \%$ and $49.7 \%$ before and after the FD implantation, respectively.

\section{Pressure}

In Case 1, the distribution of the pressure of the aneurysmal wall changed after the FD embolization. The overall pressure increased as the intra-aneurysmal pressure increased by $5.9 \%$, and the pressure of the aneurysmal wall rose by $3.5 \%$. In Case 2, there was no significant change in the pressure of the aneurysm wall after FD embolization except for a small drop. The huge difference in pressure changes predicted the converse in the treatment effect.

\section{Hemodynamics of different FDs implantation strategies}

In this part, besides the hemodynamic analysis of the aneurysm with delayed rupture before FD implantation and implanted with $30 \%$ MCR of FD (strategy A), we also added two additional FDs strategies (strategy B, FD with distal densification of $40 \%$; strategy C, FD with proximal densification of $40 \%$ ) to compare the hemodynamic effects among different FD strategies on the aneurysm with delayed rupture. Figure 5 shows the streamlines, ISO of $0.4 \mathrm{~m} / \mathrm{s}$, WSS, and the pressure of the aneurysm with delayed rupture implanted with different FD strategies. The 
Case 1

Without FD

Velocity

1.374
1.221
1.069
0.916
0.763
0.611
0.458
0.305
0.153
0.000

$\left[\mathrm{m} \cdot \mathrm{s}^{-1}\right]$

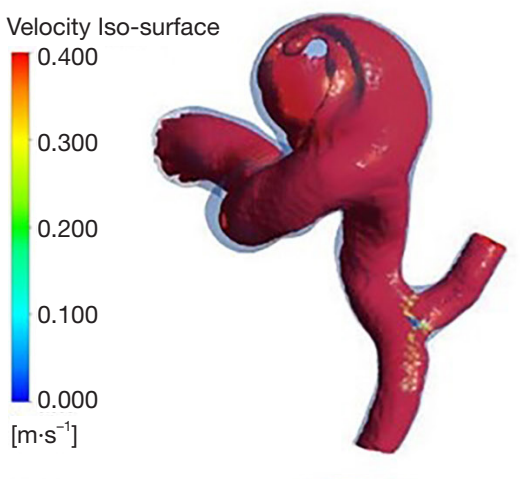

Wall shear
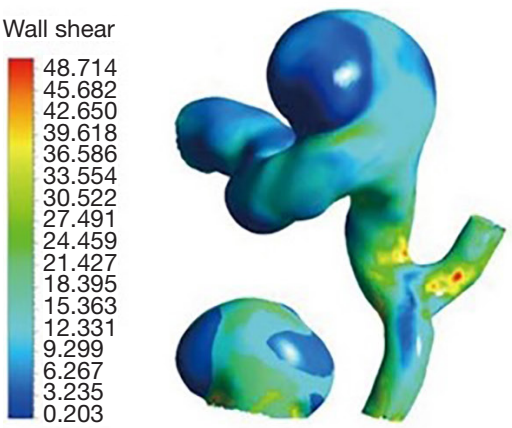

$[\mathrm{Pa}]$

Pressure

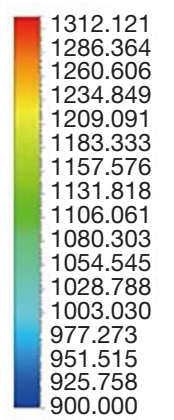

$[\mathrm{Pa}]$

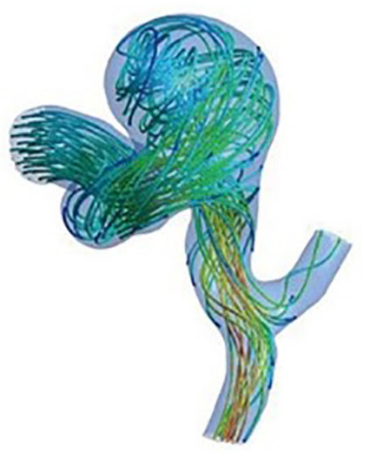

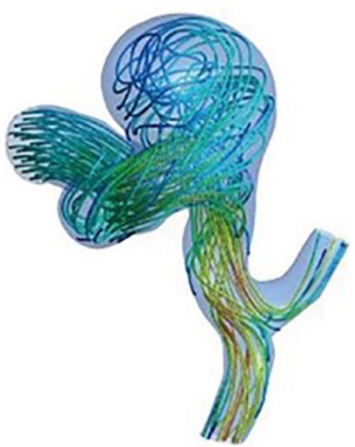
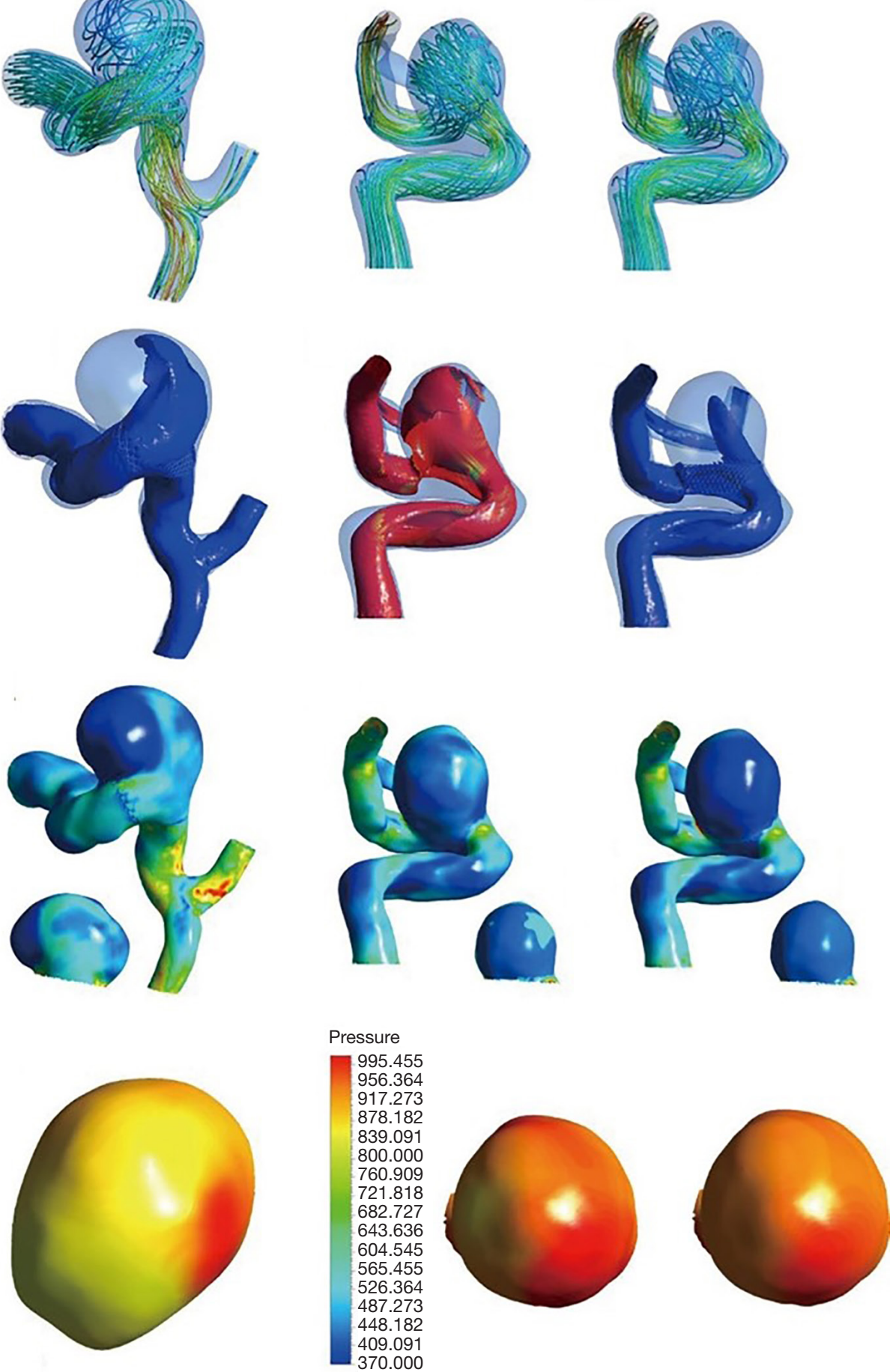

$[\mathrm{Pa}]$

Figure 3 Hemodynamics of 2 cases after flow diverters (FDs) implantation. 


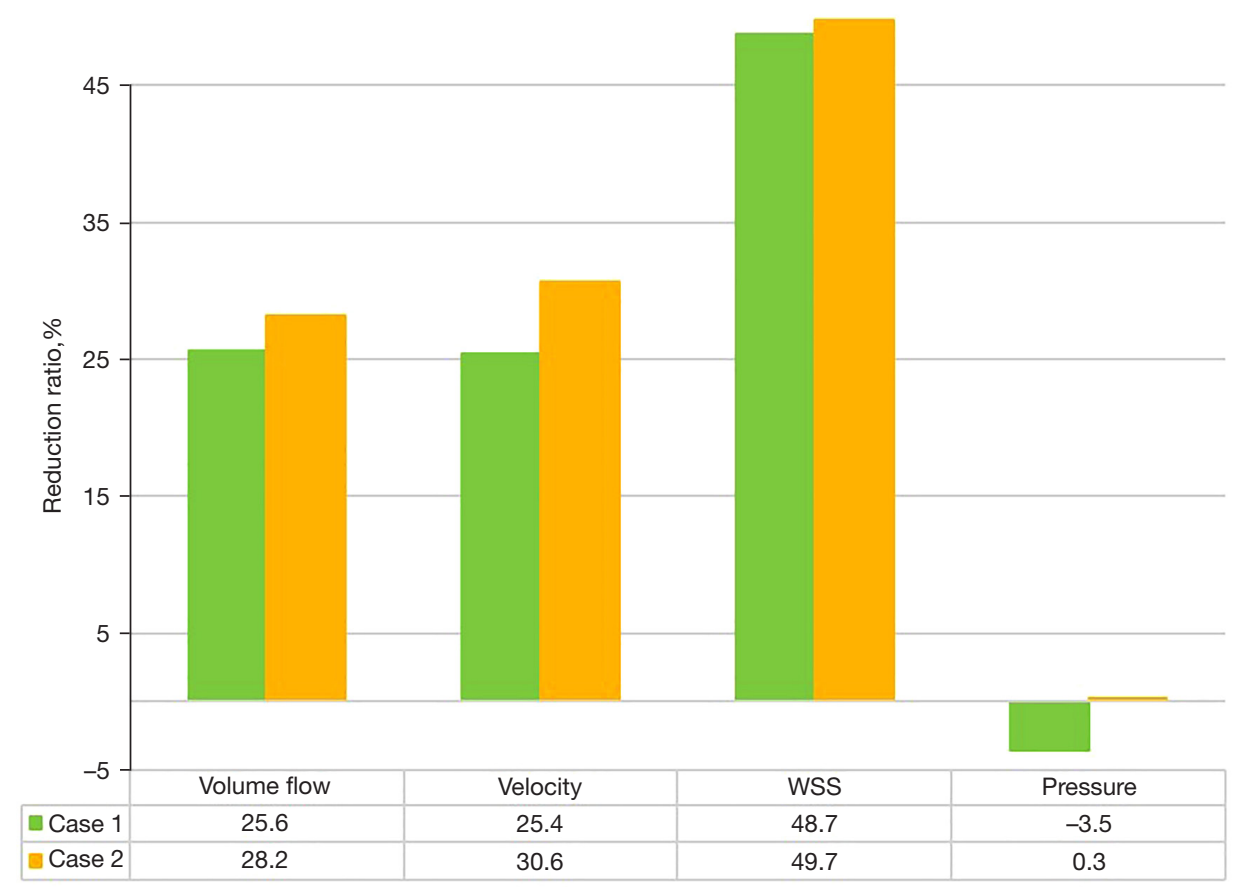

Figure 4 Comparisons of reduction ratios of hemodynamic parameters between the 2 cases.

reduction ratio of each parameter are shown in Figure 6.

\section{Velocity and volume flow of blood}

The effective reduction of the blood flow velocity is the key to aneurysm embolization. The number of streamlines and velocity of the blood flow at the aneurysm dome decreased significantly after FDs treatment. But strategy C (FD with proximal densification) had a better curative outcome for this aneurysm model with delayed rupture, not only because the blood flow vortex structure was almost disappeared and the flow pattern in the aneurysm tended to be more stable, but also the blood flow velocity at the aneurysm neck plane decreased from 0.44 to $0.21 \mathrm{~m} / \mathrm{s}$, with a decrease ratio of $52.27 \%$ which was the largest reduction among these three strategies.

\section{WSS}

The averaged WSS value of the IA was relatively high, and a comparatively large area of high WSS was observed on the aneurysm wall. After FDs treatment, the averaged WSS decreased significantly. In strategy A and strategy B, the low WSS area appeared but high WSS region still existed. In Strategy C, not only the averaged WSS decreased, but also the existing high WSS area almost disappeared, proving an excellent FD embolization.

\section{Pressure}

Before FD placement, the aneurysm wall was subjected to uneven pressure, including both the wall area with high pressure due to inflow jet impingement and the proximal wall area with low pressure due to less inflow jet impingement. After FDs implantation, it is worth nothing that in strategy A and strategy B, the overall pressure increased significantly, and the pressure increased at the low-pressure area before FD placement. However, strategy $\mathrm{C}$ with proximal densification had an encouraging outcome, as the overall pressure decreased significantly and distributed evenly.

\section{Discussion}

The rupture of IAs is catastrophic for the high morbidity and mortality. The preventive treatments include clipping surgery and endovascular treatment, both of which are effective for different types of IAs but carry non-negligible surgery-related risk at the same time. With the development of neuro-interventional imaging technology and interventional therapy devices, endovascular intervention has become the preferred treatment for its minimally invasive features. As a new endovascular tool, FDs aim to make parent artery reconstruction and aneurysm embolization by diverting the blood flow from the 
FDs treatment

Without FD

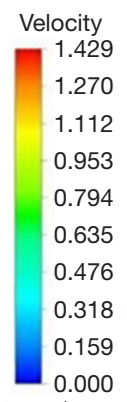

$\left[\mathrm{m} \cdot \mathrm{s}^{-1}\right]$

Velocity

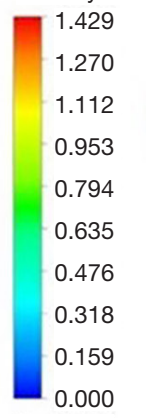

$\left[\mathrm{m} \cdot \mathrm{s}^{-1}\right]$
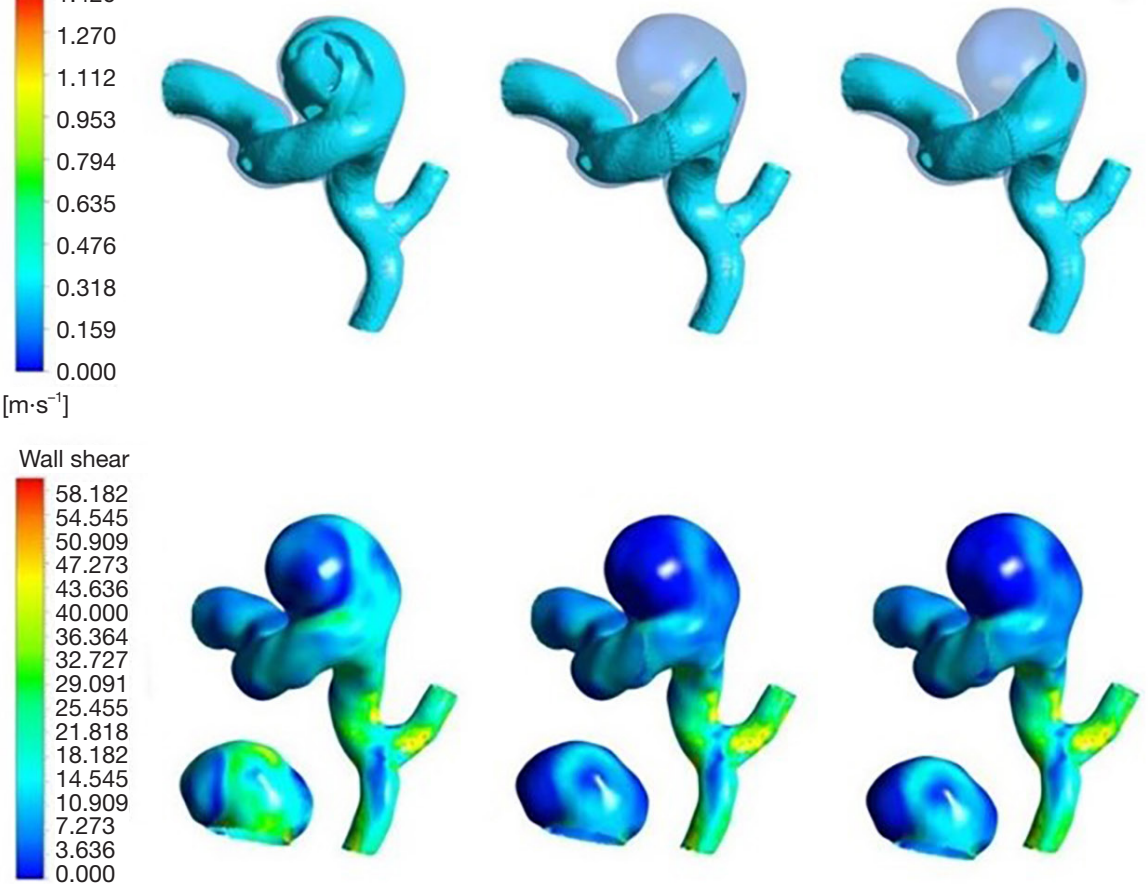

[Pa]
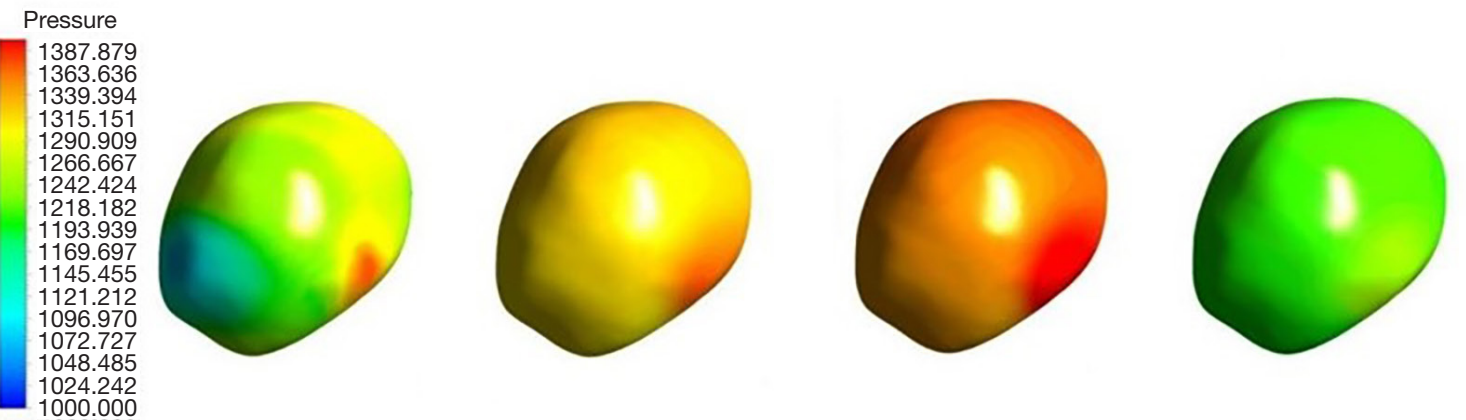

$[\mathrm{Pa}]$

Figure 5 Hemodynamics of Case 1 after different flow diverters (FDs) implantation strategies. 


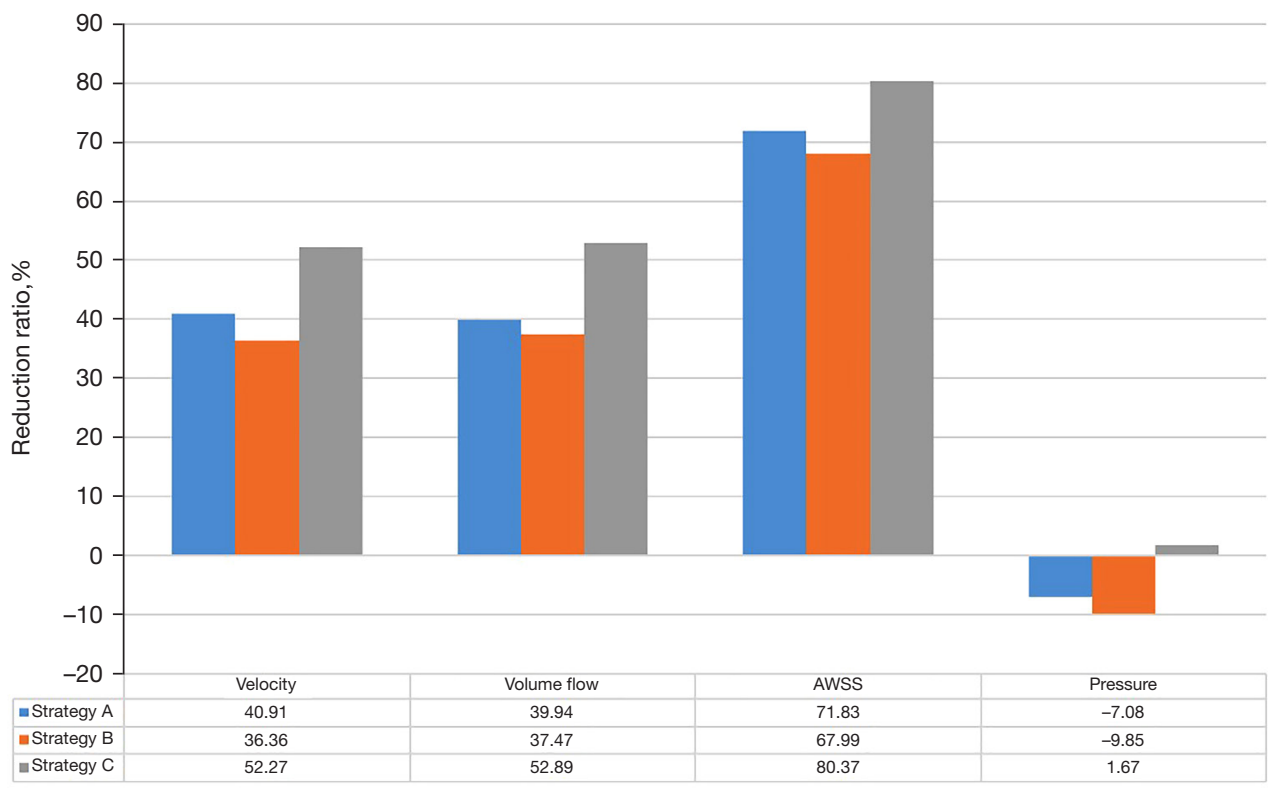

Figure 6 Comparisons of reduction ratios of hemodynamic parameters between the three different flow diverters (FDs) implantation strategies.

aneurysm sac to the parent artery, which was initially used to treat the large or giant wide-necked IAs located at the ICA proximal to the posterior communicating artery, and the curative outcomes were excellent (5). With the widely application of FDs, the surgery-related complications have been increasingly aroused our concern. Delayed aneurysm rupture is one of the most serious complications after FDs treatment, which may bring on SAH or carotid-cavernous fistula (11). Rouchaud et al. (12) reported that the prognosis of the patients with delayed aneurysm rupture was poor, with mortality or neurological dysfunction rate of $81.3 \%$. They also found that more than $80 \%$ of IAs with delayed rupture after FDs treatments were originally unruptured in their study, which was definitely unexpected. The view of FDs treatment with low MCR may not completely occlude IAs while FDs with high MCR can effectively help the reconstruction of parent artery has been supported. Nonetheless, FDs with high MCR can increase the pressure of the aneurysms by overlapping multiple FDs which may increase the rupture risk of IAs.

The selected two aneurysms in this study were located in ICA, the morphology was similar, and both patients were treated with FDs. However, they experienced entirely opposite outcomes after FDs treatment: Case 2 was significantly improved after FD treatment, while Case 1 died from delayed aneurysm rupture within 3 days. We simulated the hemodynamics before and after FDs placement (with strategy A) of two aneurysm models to analyze the hemodynamic parameters that may be associated with postoperative rupture. Afterwards, we added two additional FDs implantation strategies (strategy B, FD with distal MCR of $40 \%$ and proximal MCR of $30 \%$; strategy C, FD with proximal MCR of $40 \%$ and distal MCR of $30 \%)$ to compare the hemodynamic effects of different FD implantation strategies on Case 1 who experienced delayed aneurysm rupture.

\section{Hemodynamics of IAs after FDs implantation}

The embolization of aneurysm cannot complete immediately after FD placement, which may leave the aneurysm at risk of rupture. Chen et al. (25) suggested that reduction ratio of velocity on the neck plane of less than 0.273 and persistent inflow jet were significantly associated with the incomplete occlusion of the aneurysm after PED placement. Ouared et al. (23) including sidewall ICA or vertebral artery IAs, suggested that reduction of at least onethird in velocity entering the aneurysm after FD placement was a significant risk factor for excellent curative outcomes. In this study, there was no significant difference in the decrease of blood flow velocity between these two cases, and the blood flow velocity of Case 1 decreased by $25.38 \%$ after FD placement. However, the inflow jet impingement on the aneurysm dome still existed in Case 1, which disappeared 
in Case 2. Hence, we reckon the velocity of blood flow entering the aneurysm did not decrease effectively adding persistent impact of inflow jet impingement may cause potential aneurysm wall fragility or even aneurysm rupture, which may influence the outcome FD treatment.

WSS is the most controversial hemodynamic parameter in the mechanism of aneurysm rupture. We found that WSS of the aneurysm wall decreased, which of the vessel increased in both of the two cases after FD implantation. This changes of WSS was similar to the findings of Wang et al. (24). Meng et al. (26) supported that the rupture of IAs after FD implantation was related to the high WSS on the aneurysm wall. In this study, WSS on the aneurysm wall decreased after FD implantation in Case 1, but the distribution of high WSS area did not change. On the contrary, WSS on the aneurysm wall decreased and the originally high WSS area dissipated in Case 2. Zhang et al. (27) suggested that large areas of low WSS and small areas of high WSS on the aneurysm wall after FD implantation were the main mechanisms of rupture. We consequently consider the existence of high WSS region may influence the therapeutic outcome of FD treatments.

Pressure is a crucial parameter in the study of aneurysm hemodynamics. Suzuki et al. (28) verified that the highpressure area predicted by CFD simulations was consistent with the actual thin-walled area of the aneurysm through clipping surgery. Cebral et al. (13) studied 7 cases of aneurysms and found that in the cases of ruptured aneurysms, the pressure of the aneurysm increased, but in the successful cases, the pressure did not change significantly. After studying 3 cases of aneurysms treated by FD, Corriveau et al. (29) considered the increase of pressure caused by stent implantation was an important factor for aneurysm rupture and bleeding. In this study, we found that the pressure increased in Case 1 due to the obstructive effect of FD placement, while the pressure of Case 2 decreased, which may be a reason for the difference in the therapeutic effect of the two cases. The fragile nature of the aneurysm wall makes it susceptible to rupture under small increase pressure gradients. The significant pressure increase of the aneurysm sac in Case 1 compared with Case 2 was also reflected in the pressure distribution contour.

\section{Hemodynamics of different FDs implantation strategies}

$\mathrm{Xu}$ et al. (30) used virtual FD implantation and CFD technology, reconstructing FDs with MCR $>35 \%$ and MCR $<35 \%$ to investigate the hemodynamic effects on the aneurysm before and after FD treatments. They confirmed FDs with different MCR can affect the hemodynamic characteristics of IAs. Previous studies have mainly focused on the hemodynamic effects of FD with homogeneous MCR densification. To our knowledge, this is the first study focused on the effects of FDs with proximal densification or distal densification on the IAs before and after FD treatments.

Based on the hemodynamic characteristics related to delayed aneurysm rupture discussed above, the curative outcome of strategy $\mathrm{C}$ equipped with the $\mathrm{FD}$ with proximal MCR of $40 \%$ and distal MCR of $30 \%$ was better than the other two FD strategies. In strategy $\mathrm{C}$, the reduction ratio of blood flow velocity was the largest of $52.27 \%$, and the flow vertex in the aneurysm was almost disappeared indicating a more stable flow pattern, which can help the process of aneurysm occlusion quicker and easier after FD treatment. Furthermore, the overall WSS decreased, and the high WSS was almost disappeared which still existed whether in strategy A and strategy B. What the most interesting is that the pressure decreased only in strategy C. In view of the results above, strategy $\mathrm{C}$ was the best FD implantation among the three strategies, which can help the process of aneurysm occlusion quicker and easier to reduce the risk of delayed rupture after FD treatment. This finding can be suggestive for the clinical decision-making for the FD implantation when considering about similar cases.

Due to various assumptions and simplifications, this study had certain limitations, thus we cannot fully reveal the cause of completely different results after aneurysm FD treatment. First, the calculations in this article involved some assumptions about the boundary conditions and blood processing. Although the flow pattern of vessels exhibits non-Newtonian fluid behavior, Carty et al. (31) found that it is reasonable and feasible to set blood as Newtonian fluid in aneurysm-related research. Second, there was a certain difference between the virtual stent implantation state and the real stent implantation state in this study. Zhang et al. (32) used 3D printing technology and microCT to reconstruct the release state of the intravascular stent and compared it with the results of the virtual release stent. It was found that the distribution and magnitude of hemodynamic parameters of the 2 methods are similar and reliable. Third, the number of aneurysms in this study was too small. The identification of similar patient-specific aneurysms with different treatment outcomes is difficult. More aneurysms should be studied, and the measurements of patient-specific flow conditions should be utilized. For further research, our research group plans to address the 
above limitations, summarize more aneurysms, and use patient-specific blood flow conditions for research. We will also endeavor to incorporate 3D printing and particle image velocimetry technology, and design in vitro experiments to assist the calculation results.

\section{Conclusions}

In this study, we found that the velocity of blood flow entering the aneurysm did not decrease effectively adding persistent impact of inflow jet impingement, the existence of high WSS as well as the increase of pressure after FD placement may cause delayed aneurysm rupture. In addition, the hemodynamic changes were favorable when the FD was improved to proximal densification which may reduce the risk of delayed aneurysm rupture following FD treatment.

\section{Acknowledgments}

Funding: This work was supported by the Shanghai Municipal Health Commission's Special Plan for Clinical Research in the Health Industry (20194Y0131), the National Natural Science Foundation of China Youth Project (81701775) and the Top-level Clinical Discipline Project of Shanghai Pudong (PWYgf2021-07).

\section{Footnote}

Reporting Checklist: The authors have completed the MDAR reporting checklist. Available at https://dx.doi. org/10.21037/atm-21-5939

Data Sharing Statement: Available at https://dx.doi. org/10.21037/atm-21-5939

Conflicts of Interest: All authors have completed the ICMJE uniform disclosure form (available at https://dx.doi. org/10.21037/atm-21-5939). The authors have no conflicts of interest to declare.

Ethical Statement: The authors are accountable for all aspects of the work in ensuring that questions related to the accuracy or integrity of any part of the work are appropriately investigated and resolved. The study was conducted in accordance with the Declaration of Helsinki (as revised in 2013). This retrospective study was approved by the Institution Review Board of Changhai Hospital. The requirement for informed consent was waived for this retrospective study.

Open Access Statement: This is an Open Access article distributed in accordance with the Creative Commons Attribution-NonCommercial-NoDerivs 4.0 International License (CC BY-NC-ND 4.0), which permits the noncommercial replication and distribution of the article with the strict proviso that no changes or edits are made and the original work is properly cited (including links to both the formal publication through the relevant DOI and the license). See: https://creativecommons.org/licenses/by-nc-nd/4.0/.

\section{References}

1. Brisman JL, Song JK, Newell DW. Cerebral aneurysms. N Engl J Med 2006;355:928-39.

2. Connolly ES Jr, Rabinstein AA, Carhuapoma JR, et al. Guidelines for the management of aneurysmal subarachnoid hemorrhage: a guideline for healthcare professionals from the American Heart Association/ american Stroke Association. Stroke 2012;43:1711-37.

3. Gory B, Berge J, Bonafé A, et al. Flow Diverters for Intracranial Aneurysms: The DIVERSION National Prospective Cohort Study. Stroke 2019;50:3471-80.

4. Zhu D, Yan Y, Zhao P, et al. Safety and Efficacy of Flow Diverter Treatment for Blood Blister-Like Aneurysm: A Systematic Review and Meta-Analysis. World Neurosurg 2018;118:e79-86.

5. Chancellor B, Raz E, Shapiro M, et al. Flow Diversion for Intracranial Aneurysm Treatment: Trials Involving Flow Diverters and Long-Term Outcomes. Neurosurgery 2020;86:S36-45.

6. Lylyk P, Miranda C, Ceratto R, et al. Curative endovascular reconstruction of cerebral aneurysms with the pipeline embolization device: the Buenos Aires experience. Neurosurgery 2009;64:632-42; discussion 642-3; quiz N6.

7. Tomasello A, Romero N, Aixut S, et al. Endovascular treatment of intracraneal aneurysm with pipeline embolization device: experience in four centres in Barcelona. Neurol Res 2016;38:381-8.

8. Brinjikji W, Murad MH, Lanzino G, et al. Endovascular treatment of intracranial aneurysms with flow diverters: a meta-analysis. Stroke 2013;44:442-7.

9. Kallmes DF, Hanel R, Lopes D, et al. International retrospective study of the pipeline embolization device: a multicenter aneurysm treatment study. AJNR Am J Neuroradiol 2015;36:108-15.

10. Zhou G, Su M, Yin YL, et al. Complications associated 
with the use of flow-diverting devices for cerebral aneurysms: a systematic review and meta-analysis. Neurosurg Focus 2017;42:E17.

11. Rajah G, Narayanan S, Rangel-Castilla L. Update on flow diverters for the endovascular management of cerebral aneurysms. Neurosurg Focus 2017;42:E2.

12. Rouchaud A, Brinjikji W, Lanzino G, et al. Delayed hemorrhagic complications after flow diversion for intracranial aneurysms: a literature overview. Neuroradiology 2016;58:171-7.

13. Cebral JR, Mut F, Raschi M, et al. Aneurysm rupture following treatment with flow-diverting stents: computational hemodynamics analysis of treatment. AJNR Am J Neuroradiol 2011;32:27-33.

14. Tateshima S, Jones JG, Mayor Basto F, et al. Aneurysm pressure measurement before and after placement of a Pipeline stent: feasibility study using a 0.014 inch pressure wire for coronary intervention. J Neurointerv Surg 2016;8:603-7.

15. Xiang J, Damiano RJ, Lin N, et al. High-fidelity virtual stenting: modeling of flow diverter deployment for hemodynamic characterization of complex intracranial aneurysms. J Neurosurg 2015;123:832-40.

16. Szikora I, Berentei Z, Kulcsar Z, et al. Treatment of intracranial aneurysms by functional reconstruction of the parent artery: the Budapest experience with the pipeline embolization device. AJNR Am J Neuroradiol 2010;31:1139-47.

17. Roszelle BN, Gonzalez LF, Babiker MH, et al. Flow diverter effect on cerebral aneurysm hemodynamics: an in vitro comparison of telescoping stents and the Pipeline. Neuroradiology 2013;55:751-8.

18. Nelson PK, Lylyk P, Szikora I, et al. The pipeline embolization device for the intracranial treatment of aneurysms trial. AJNR Am J Neuroradiol 2011;32:34-40.

19. Kulcsár Z, Houdart E, Bonafé A, et al. Intra-aneurysmal thrombosis as a possible cause of delayed aneurysm rupture after flow-diversion treatment. AJNR Am J Neuroradiol 2011;32:20-5.

20. Lieber BB, Stancampiano AP, Wakhloo AK. Alteration of hemodynamics in aneurysm models by stenting: influence of stent porosity. Ann Biomed Eng 1997;25:460-9.

21. Wiebers DO, Whisnant JP, Huston J 3rd, et al.

Unruptured intracranial aneurysms: natural history, clinical outcome, and risks of surgical and endovascular treatment. Lancet 2003;362:103-10.

22. Vercelli G, Sorenson TJ, Aljobeh AZ, et al. Cavernous sinus aneurysms: risk of growth over time and risk factors.
J Neurosurg 2019;132:22-6.

23. Ouared R, Larrabide I, Brina O, et al. Computational fluid dynamics analysis of flow reduction induced by flow-diverting stents in intracranial aneurysms: a patient-unspecific hemodynamics change perspective. J Neurointerv Surg 2016;8:1288-93.

24. Wang C, Tian Z, Liu J, et al. Hemodynamic alterations after stent implantation in 15 cases of intracranial aneurysm. Acta Neurochir (Wien) 2016;158:811-9.

25. Chen J, Zhang Y, Tian Z, et al. Relationship between haemodynamic changes and outcomes of intracranial aneurysms after implantation of the pipeline embolisation device: a single centre study. Interv Neuroradiol 2019;25:671-80.

26. Meng H, Tutino VM, Xiang J, et al. High WSS or low WSS? Complex interactions of hemodynamics with intracranial aneurysm initiation, growth, and rupture: toward a unifying hypothesis. AJNR Am J Neuroradiol 2014;35:1254-62.

27. Zhang Y, Jing L, Zhang Y, et al. Low wall shear stress is associated with the rupture of intracranial aneurysm with known rupture point: case report and literature review. BMC Neurol 2016;16:231.

28. Suzuki T, Takao H, Suzuki T, et al. Determining the Presence of Thin-Walled Regions at High-Pressure Areas in Unruptured Cerebral Aneurysms by Using Computational Fluid Dynamics. Neurosurgery 2016;79:589-95.

29. Corriveau M, Ahmed A, Dawkins D, et al. The effect of flow diverting stents on in vivo intrasaccular aneurysm pressure. J Clin Neurosci 2019;59:339-41.

30. Xu J, Deng B, Fang Y, et al. Hemodynamic Changes Caused by Flow Diverters in Rabbit Aneurysm Models: Comparison of Virtual and Realistic FD Deployments Based on MicroCT Reconstruction. PLoS One 2013;8:e66072.

31. Carty G, Chatpun S, Espino DM. Modeling blood flow through intracranial aneurysms: A comparison of Newtonian and non-Newtonian viscosity. Journal of Medical and Biological Engineering 2016;36:396-409.

32. Zhang Q, Liu J, Zhang Y, et al. Efficient simulation of a low-profile visualized intraluminal support device: a novel fast virtual stenting technique. Chin Neurosurg J 2018;4:6.

(English Language Editor: J. Jones)

Cite this article as: Chen S, Bai B, Lv N, Cheng Y, Ji B. Hemodynamic analysis and implantation strategies of delayed intracranial aneurysm rupture after flow diverter treatment. Ann Transl Med 2021;9(23):1735. doi: 10.21037/atm-21-5939 